\title{
RECHERCHES EXPÉRIMENTALES SUR LA TOXICITÉ DU FLUOR POUR LES ABEILLES
}

\author{
C. TOUMANOFF \\ Institut Pasteur, Paris.
}

SOMMAIRE

Les expériences qui sont relatées dans ce travail ont démontré que le fluor (sous forme de $\mathrm{NaF}$ en solution dans un sirop sucré) n'est toxique pour les abeilles qu'à des concentrations trop élevées pour qu'on puisse les voir se réaliser dans la nature.

Les abeilles nourries au moyen d'un sirop contenant du fluor en faible proportion peuvent ingérer des quantités de cet élément supérieures à celles qui sont considérées par certains auteurs comme doses léthales et elles présentent souvent la même survie que les abeilles témoins.

Une des modalités de nourrissement des abeilles décrite dans ce travail a permis de constater la possibilité d'une accoutumance des abeilles à une dose normalement mortelle de fluor.

\section{IN'TRODUC'TION}

Il est bien connu que quelques produits fluorés exercent un effet toxique sur les insectes et que leurs propriétés ont même été utilisées pour la lutte contre certains insectes nuisibles.

Toutefois, si 1'on en juge par les faits, et selon les considérations générales rapportées à ce sujet, entre autres par BEI-BIENko et al. (I955) dans leur ouvrage d'Entomologie agricole, l'application des produits fluorés se heurte à des difficultés en fonction des conditions extérieures, de la nature et de la composition des produits, de leur solubilité, et aussi quoique cela soit moins bien établi, de l'état physiologique de l'insecte que l'on veut atteindre. Il réstulte des observations de nombreux auteurs que, dans l'ensemble les produits fluorés sont bien moins efficaces contre les insectes que les arsénicaux et les insecticides organiques de contact.

Depuis longtemps, déjà, les dégagements fluorés des usines préparant l'aluminium et ceux d'autres industries, ont été considérés comme étant la cause d'intoxications d'abeilles. Nous avons eu l'occasion de traiter cette question dans notre ouvrage sur les maladies des abeilles (ToumanofF C., I95I), mais, à 1'époque, notre expérience personnelle sur ce sujet n'était que rudimentaire et nous nous sommes contentés de relater fidèlement les indications bibliographiques. 
Les enquêtes que nous avons effectuées sur le terrain tant en France qu'en Autriche nous ont convaincu que la forte toxicité des émanations fluorées des usines pour les abeilles, invoquéa par divers auteurs (BORCHER'T, I953; BREDEMANN I956, Bredemani et RadeiofF i 939, Gullhon i956, Maurizio i 955, Maurizio et S'TAub I956, Maurizio I957, Rousseau I954, I957) est fortement exagérée, et parfois sujette à caution. Nous avons indiqué dans plusieurs publications antérieures que, chez les abeilles provenant de richers se trouvant près d'usines préparant l'aluminium aussi bien en Autriche qu'en France, le fluor était décelé individuellement en très grandes quantités, quantités supérieures à celle considérée par certains auteurs. comme "dose léthale " (JACHIMOWICZ, Toumanoff et WeII.ER, I958).

Cette cirsconstance nous a incités à effectuer des expériences sur l'action du fluor sous forme de $\mathrm{NaF}$ sur les abeilles en administrant à des colonies contenues dans des ruchettes ou dans des ruches, un sirop de sucre contenant du fluor à des concentrations variées. (Expériences réalisées en collaboration avec JACHIMOWICZ en Autriche, en France et Toumanoff et Jachimowicz 1959-I960, Toumanoff ig6r.)

Nos essais nous ont conduit à cette conclusion qu'il est pratiquement impossible de faire périr les colonies contenues dans les ruches ou les ruchettes en leur fournissant. un sirop fluoré.

Nous avons conclu aussi que l'établissement de la dose "léthale " par une analyse chimique ne donne pas de résultats concluants car chez les abeilles saines le fluor a été décelé en quantités correspondant à la dose léthale ou la dépassant.

Il nous a paru intéressant de connaitre la quantité de fluor que peut absorber une abeille lorsqu'on lui fournit du sirop de sucre ou du miel additionné de $\mathrm{NaF}$ en quantité donnée, c'est-à-đire contenant du fluor dans les proportions de $\mathrm{I} / \mathrm{I}$ ooo, I $/ 2$ ooo, etc.

Ces expériences n'ont pas revêtu, comme on le verra, un caractère uniforme.

\section{MÉTHODES EXPÉRIMENTALES E'T MATÉRIEL U'TILISÉ}

Les abeilles qui ont servi pour nos essais ont été prélevées dans les ruches du Rucher expérimental de notre Service à l'Institut Pasteur.

Dans les expériences que nous avons effectuées, nous avons utilisé des groupes d'abeilles prélevées. dans les ruches en quantités variables et placées dans de petites cagettes en bois munies par devant d'une vitre et comportant à leur partie supérieure deux trous destinés à recevoir des tubes gradués. percés à leur partie inférieure d'un petit trou par lequel les abeilles absorbent le sirop. Lorsque le tube est bien rempli et fermé, il ne laisse pas écouler le liquide sucré.

Ces cagettes ne sont pas notre invention ; nous nous sommes inspirés de celles que nous avons. vues chez le prof. Goetze en Allemagne, à Bonn.

Nous avons ainsi cherché à connaitre la quantité de fluor consommé par des lots d'abeilles maintenus en cagette (et aussi individuellement par chaque abeille) en évaluant la quantité de solution fluorée de concentration connue consommée par lot d'abeilles et par individu.

Il est possible, bien entendu, que toutes les abeilles du même lot n'aient pas le même appétit et qu'elles n'aient pas toutes absorbé la même quantité de nourriture, ni par conséquent la même quantité de fluor. Néanmoins, une chose demeure certaine, c'est qu'elles s'attaquent toutes à la nourriture qui leur est offerte, non pas contraintes et forcées, mais librement.

Cette manière de procéder est certainement meilleure que l'administration individuelle aux abeilles, tenues par les ailes, de sirop fluoré avec une pipette qu'on présente devant leur trompe, procédé plutôt brutal proposé par certains auteurs et qu'on ne saurait accepter dans la pratique étant donné qu'il s'accompagne d'un traumatisme.

Si le procédé expérimental que nous avons adopté n'est pas totalement à l'abri des critiques, c'est cependant le meilleur qu'on puisse envisager. 


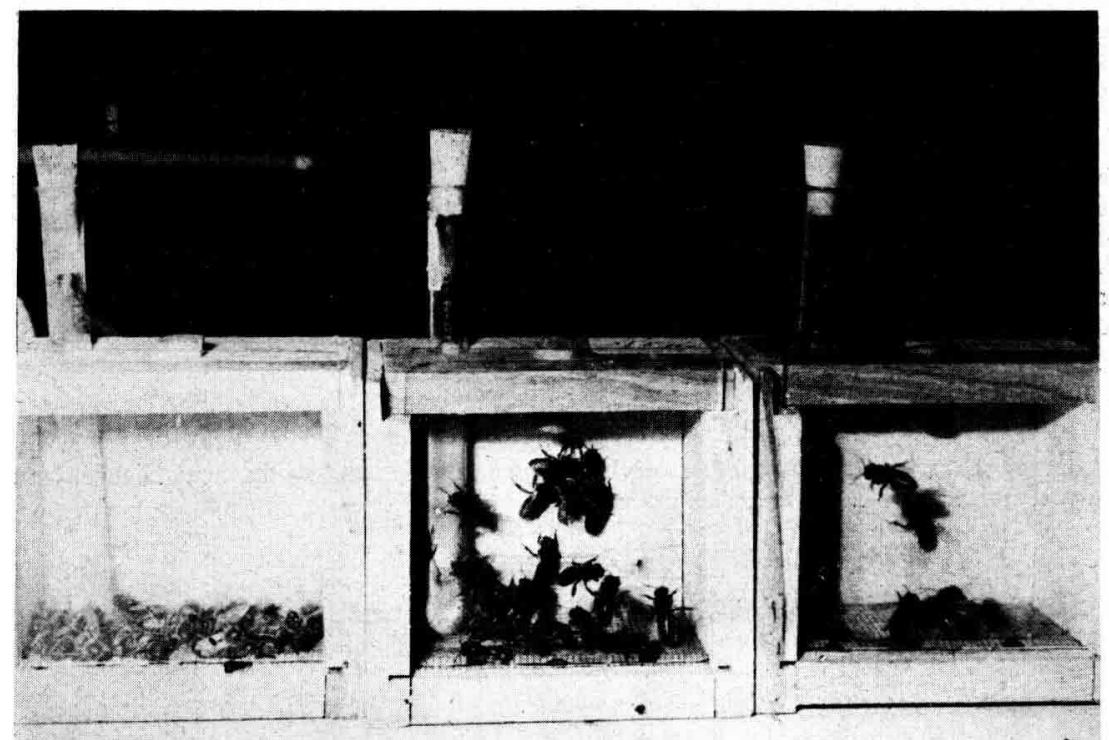

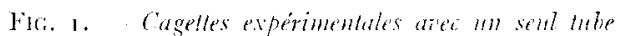

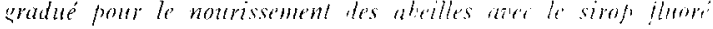

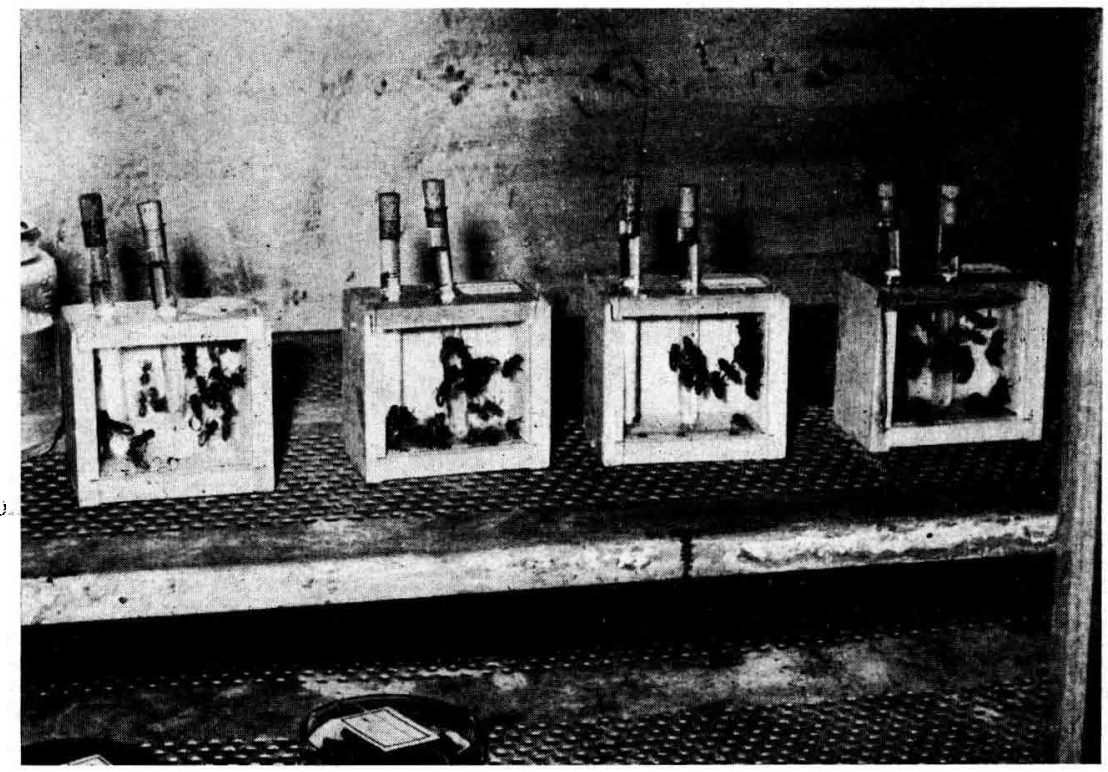

Fis. 2. - Quelutes cagettes expérimentales mumies des lem tulies gradués:

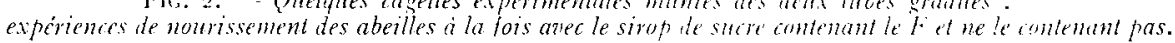


Dans ce premier exposé, nous allons relater les résultats de nos essais d'intoxication d'abeilles par des solutions plus ou moins concentrées de $\mathrm{NaF}$.

Nous avons enregistré journellement l'effet des solutions fluorées sur les abeilles et les résultats obtenus ont été portés sur des tableaux, qui en raison de leur longueur ne pouvaient pas êtreimprimés. C'est pourquoi nous avons adopté un système de présentation des résultats qui consiste à ne pas donner tous les détails concernant les mortalités journalières mais à indiquer la survie moyenne des abeilles dans chaque expérience d'administration de sirop fluoré aux abeilles. Sur ce tableaux figurent également les indications sur la consommation totale de sirop pendant la durée de l'expérience, la consommation en fluor par abeille et par jour (en $\gamma$ ) et aussi la consommation totale de fluor (en $\gamma$ ) pendant une survie moyenne. La survie moyenne des témoins est également rapportée sur ce tableau. Nous avons enfin ajouté à ce tableau des indications sur la survie maxima des abeilles au cours de chacune de nos expériences.

Afin de rendre la lecture de nos tableaux plus aisée, nous présentons ci-dessous les explications concernant la base des calculs qui ont été effectués pour leur établissement.

$1^{\circ}$ Calcul de la survie moyenne d'un lot d'abeilles.

On compte chaque jour le nombre de survivants et on fait le total en fin d'expérience. On divise le chiffre obtenu par le nombre d'abeilles du lot.

$2^{\circ}$ Calcul de la dose journalière de sirop par abeille.

On divise la quantité de sirop consommée au cours de l'expérience par le nombre total de survies, calculé précédemment (le chiffre est exprimé en cc).

$3^{\circ}$ Calcul de la dose journalière de fuor par abeille.

On divise le chiffre précédent par celui de la dilution expérimentale (le chiffre est exprimé en $\gamma$ ).

$4^{\circ}$ Calcul de la dose totale de fuor consommé par une abeille de survie moyenne.

On multiplie le chiffre précédent par celui de la durée moyenne de vie.

Les résultats de nos essais effectués avec le sirop de sucre fluoré sont les seuls présentés dans ce travail ; nous envisageons de rapporter ultérieurement ceux qui ont été obtenus avec un sirop miellé.

Chaque tableau comporte une série d'expériences ainsi que les témoins.

\section{RÉSULTATS}

\section{I' L'EFFET DU FLUUOR SUR LES ABEILLES A DES CONCENTRATIONS DIVERSES}

\section{Série A}

(Commencée le 9 janvier, terminée le 29 janvier.)

Comme on peut le voir en examinant ce tableau la survie maxima a varié dans les six expériences composant cette série de 5 à 19 jours. Les abeilles qui ont reçu le sirop de sucre dont la concentration était de I/I5 000 à $\mathrm{I} / 25000$ sont mortes plus rapidement que celles qui ont été alimentées par les sirops à la concentration moins forte de $1 / 25000$ à I/I00 000 . On constate même que la survie maxima des abeilles qui ont été alimentées par les sirops contenant respectivement : I/30 000, I/50 ooo et I/ ro ooo de fluor fut supérieure à celle des lots témoins nourris au sirop de sucre pur La survie moyenne des abeilles soumises à l'intoxication par les trois sirops mentionnés était supérieure ou comparable à celle des lots témoins.

On remarque, par ailleurs, que les lots d'abeilles soumises à l'intoxication consommèrent pendant la période de leur survie de 7 à $2 \mathrm{I} \gamma$ de fluor par abeille et que celles qui furent nourries au sirop de sucre à $\Upsilon / 50$ ooo ont présenté la survie maxima et une survie moyenne supérieure à celle de tous les autres lots. 
TABLEAU I

\begin{tabular}{|c|c|c|c|c|c|c|c|}
\hline No Expérience & $\begin{array}{l}\text { Nombre } \\
\text { d'abeilles }\end{array}$ & $\mid \begin{array}{c}\text { Consomma- } \\
\text { tion de } \\
\text { sirop (en cc) }\end{array}$ & $\begin{array}{l}\text { Concentr. } \\
\text { du sirop } \\
\text { en fluor }\end{array}$ & $\begin{array}{c}\text { Survie } \\
\text { maximum } \\
\text { (en jours) }\end{array}$ & $\begin{array}{l}\text { Survie } \\
\text { moyenne } \\
\text { (en jours) }\end{array}$ & $\begin{array}{l}\text { Consomma- } \\
\text { tion en } \\
\text { F/Ab/jour } \\
(\mathrm{en} \gamma)\end{array}$ & $\begin{array}{c}\text { Consomm } \\
\text { totale F/Ab } \\
\text { (surv. moy.) } \\
(\mathrm{en} \gamma)\end{array}$ \\
\hline $\begin{array}{l}1 \ldots \ldots \ldots \ldots \ldots \\
2 \ldots \ldots \ldots \ldots \ldots \\
3 \ldots \ldots \ldots \ldots \\
4 \ldots \ldots \ldots \\
5 \ldots \ldots \ldots \ldots \\
6 \ldots \ldots \ldots\end{array}$ & $\begin{array}{r}17 \\
13 \\
9 \\
10 \\
10 \\
15\end{array}$ & $\begin{array}{l}4,9 \\
5,5 \\
2,9 \\
6,5 \\
8 \\
10\end{array}$ & $\begin{array}{l}1 / 15000 \\
1 / 20000 \\
1 / 25000 \\
1 / 30000 \\
1 / 50000 \\
1 / 100000\end{array}$ & $\begin{array}{r}9 \\
10 \\
5 \\
17 \\
19 \\
17\end{array}$ & $\begin{array}{r}4,3 \\
4,8 \\
3 \\
7,8 \\
12,1 \\
9,5\end{array}$ & $\begin{array}{l}4,5 \\
4,3 \\
4,3 \\
2,7 \\
1,3 \\
0,71\end{array}$ & $\begin{array}{r}19 \\
21 \\
13 \\
21 \\
16 \\
7\end{array}$ \\
\hline $\mathrm{T} 1 \ldots \ldots \ldots \ldots \ldots$ & $\begin{array}{r}8 \\
14\end{array}$ & $\begin{array}{l}10 \\
10\end{array}$ & & $\begin{array}{l}16 \\
15\end{array}$ & $\begin{array}{l}9,6 \\
7,9\end{array}$ & & \\
\hline
\end{tabular}

\section{Série B}

Les expériences de cette série furent effectuées dans les mêmes conditions que celles de la série précédente mais plus tardivement, c'est-à-dire entre le $I_{7}$ janvier et le 7 mars.

Les résultats obtenus sont plus réguliers que dans les essais précédents. En effet, la survie maxima ainsi que la survie moyenne des abeilles augmentent proportionnellement, à une exception près, à la diminution de la concentration du fluor dans le sirop administré, ainsi qu'on peut le constater à l'examen du tableau 2 .

TABLEAU 2

\begin{tabular}{|c|c|c|c|c|c|c|c|}
\hline No Expérience & $\begin{array}{l}\text { Nombre } \\
\text { d'abeilles }\end{array}$ & $\begin{array}{c}\text { Consomma- } \\
\text { tion de } \\
\text { sirop } \\
\text { (en cc) }\end{array}$ & $\begin{array}{l}\text { Concentra- } \\
\text { tion du } \\
\text { sirop en } \\
\text { fluor }\end{array}$ & $\begin{array}{c}\text { Survie } \\
\text { maximum } \\
\text { (en jours) }\end{array}$ & $\begin{array}{c}\text { Survie } \\
\text { moyenne } \\
\text { (en jours) }\end{array}$ & $\begin{array}{c}\text { Consomma- } \\
\text { tion en } \\
\text { F/Ab/jour } \\
\text { (en } \gamma)\end{array}$ & $\begin{array}{l}\text { Consomm. } \\
\text { tot. F/Ab } \\
\text { (surv. moy.) } \\
\text { (en) } \gamma\end{array}$ \\
\hline $7 \ldots \ldots \ldots \ldots$ & 11 & 3,7 & $1 / 15000$ & 12 & 7,2 & 3,1 & 22 \\
\hline $8 \ldots \ldots \ldots \ldots$ & 12 & 5,8 & 1) 20000 & 28 & 18,8 & 1,3 & 24 \\
\hline $9 \ldots \ldots \ldots \ldots$ & 13 & 7,4 & 1) 25000 & 18 & 13 & 1,7 & 22 \\
\hline $10 \ldots \ldots \ldots \ldots$ & 7 & 6,8 & 1) 30000 & 28 & 22 & 1,4 & 31 \\
\hline $11 \ldots \ldots \ldots \ldots$ & 8 & 8,6 & 1) 50000 & 30 & 25,3 & 0,84 & 21 \\
\hline $12 \ldots \ldots \ldots \ldots \ldots$ & 9 & 14,8 & $1 / 100000$ & 50 & 35,6 & 0,46 & 16 \\
\hline $\mathrm{T} 1 \ldots$ & 9 & 7,5 & & 16 & 10,9 & & \\
\hline $\mathrm{T} 2 \ldots \ldots \ldots$ & 9 & 12,6 & & 35 & 18,4 & & \\
\hline
\end{tabular}

Ce qu'il est intéressant de noter, c'est que les abeilles des lots qui reçurent les sirops à la concentration de $\mathrm{I} / 30000, \mathrm{I} / 50$ ooo et $\mathrm{I} / \mathrm{I}$ / 00 ooo ont présenté une longévité moyenne 2,5 à 3 fois plus grande que celle des abeilles des lots témoins.

On observe, ici, non setulement l'absence d'intoxication des abeilles, mais leur vitalité plus grande sous l'influence d'une addition de fluor. Elles consomment dans ces trois lots expérimentaux 31,21 et $16 \gamma$ de fluor par abeilles, quantités de 3 à 6 fois plus fortes que celles qui sont considérées comme leur dose léthale par certains auteurs. 


\section{Séries $C$ et $D$}

Ces séries, composées repectivement de 7 et 5 expériences ont revêtı le même caractère que les deux précédentes. Les abeilles ont reçu les mêmes concentrations de fluor, la série C étant réalisée entre le 23 janvier et le I 8 mars, la série $D$ entre le 7 février et le 21 mars.

Il serait fastidieux et inutile de donner les détails de ces expériences et de leurs résultats que le lecteur suivra sur les tableaux 3 et 4 . Notons seulement que dans la série $\mathrm{C}$, la survie maxima du témoin fut légèrement plus grande que celle des abeilles qui reçurent le sirop fluoré à I/Ioo ooo. Par contre, la survie moyenne fut nettement plus élevée pour les lots témoins que pour ceux intoxiqués par cette faible dose de fluor.

TABLEAU 3

\begin{tabular}{|c|c|c|c|c|c|c|c|}
\hline No Expérience & $\begin{array}{l}\text { Nombre } \\
\text { dabeilles }\end{array}$ & $\begin{array}{l}\text { Consomma- } \\
\text { tion de } \\
\text { sirol) } \\
\text { (en cc) }\end{array}$ & $\begin{array}{l}\text { Concentr. } \\
\text { du sirop } \\
\text { en fuor }\end{array}$ & $\begin{array}{c}\text { Survie } \\
\text { maximum } \\
\text { (en jours) }\end{array}$ & $\begin{array}{c}\text { Survie } \\
\text { moyenue } \\
\text { (en jours) }\end{array}$ & $\begin{array}{c}\text { Consomma- } \\
\text { tion en } \\
\text { F/Ab/jour } \\
(\mathrm{en} \gamma)\end{array}$ & $\begin{array}{l}\text { Consomm. } \\
\text { tot. F/Ab } \\
\text { (surv.moy.) } \\
\text { (en) } \gamma\end{array}$ \\
\hline $13 \ldots \ldots \ldots \ldots$ & 19 & 3,75 & 1) 15000 & 10 & 5,8 & 2,2 & $1: 3$ \\
\hline $14 \ldots \ldots \ldots \ldots \ldots$ & 13 & 4,3 & 1/ 20000 & 10 & 7,4 & 2,2 & 16 \\
\hline $15 \ldots \ldots \ldots \ldots$ & .. 9 & 6,2 & 1) 25000 & 29 & 16,5 & 1,6 & 26 \\
\hline $16 \ldots \ldots \ldots \ldots$ & $!$ & 7,2 & 1) 30000 & 19 & 12,8 & 2,1 & 27 \\
\hline $17 \ldots \ldots \ldots \ldots$ & 11 & 7,8 & 1/ 50000 & $\because 0$ & 14,9 & 0,96 & $1 \%$ \\
\hline $18 \ldots \ldots \ldots \ldots$ & 11 & 18,1 & $1 / 100000$ & 52 & $-9,2$ & 0,56 & 16 \\
\hline Témoin ......... & 15 & 30,3 & & 54 & 39,3 & & \\
\hline
\end{tabular}

TABLEAU 4

\begin{tabular}{|c|c|c|c|c|c|c|c|}
\hline No lexpérience & $\begin{array}{l}\text { Nombre } \\
\text { d'abeilles }\end{array}$ & $\begin{array}{l}\text { Consomma- } \\
\text { tion de } \\
\text { sirop (en cc) }\end{array}$ & $\begin{array}{l}\text { Concentra- } \\
\text { tion dusirop } \\
\text { en fluor }\end{array}$ & $\begin{array}{c}\text { Survie } \\
\text { maximum } \\
\text { (en jours) }\end{array}$ & $\begin{array}{c}\text { Survic } \\
\text { moyenne } \\
\text { (en jours) }\end{array}$ & $\begin{array}{l}\text { Consomma- } \\
\text { tion en } \\
\mathrm{J} / \mathrm{Ab} / \text { jour } \\
(\mathrm{en} \gamma)\end{array}$ & $\begin{array}{c}\text { Consomm. } \\
\text { totale } \mathrm{F} / \mathrm{Ab} \\
\text { (surv. moy.) } \\
(\text { en } \gamma)\end{array}$ \\
\hline $19 \ldots \ldots \ldots \ldots$ & 12 & 7,2 & 1/ 15000 & 20 & 17,6 & 2,3 & 40 \\
\hline $20 \ldots \ldots \ldots \ldots$ & 13 & $j$ & 1) 20000 & 15 & 12 & 1,6 & 19 \\
\hline $21 \ldots \ldots \ldots \ldots \ldots$ & 16 & 9,2 & $1 / 25000$ & 23 & 15,5 & 1,5 & 23 \\
\hline $22 \ldots \ldots \ldots \ldots$ & 13 & $10, \overline{5}$ & $1 / 30000$ & 22 & 15,9 & 1,7 & 27 \\
\hline $23 \ldots \ldots \ldots \ldots$ & 22 & 21 & 1) 50000 & 85 & $2^{\prime}+2$ & 0,78 & 19 \\
\hline $24 \ldots \ldots \ldots \ldots$ & 9 & 11,9 & $1 / 100000$ & 42 & 32,4 & 0,41 & 13 \\
\hline Témoin ........ & $\therefore$ & 10,2 & i & 12 & $2 ;, 5$ & & \\
\hline
\end{tabular}

Par contre, dans les essais de la série D la survie maxima et la survie moyenne des abeilles qui reçurent le sirop à I/I oo ooo de fluor, fut identique à celle des témoins la survie moyenne étant cependant supérieure pour le lot d'Abeilles intoxiquées à cette faible dose. Dans cet essai, la survie moyenne des abeilles témoins correspondait à celle des abeilles qui reçurent le fluor à $\mathrm{I} / 50$ ooo et qui en absorbèrent en moyenne r $\gamma$ par individu. 


\section{Séries $E$ et $F$}

Ces séries diffèrent des précédentes par le fait, que la série E était commencée à 1a fin du mois de mars et terminée au début du mois de mai, plus précisément entre le 26-3 et 1e 5-5, et la sétie $F$ commencée le $I_{5}$ juin et terminée le 6 septembre.

Dans la série $E$, les solutions du fluor variaient de $I / I$ ooo à $I / I 00000$, tandis que dans la série $\mathrm{F}$ ce sont surtout les concentrations fortes qui ont été administrées aux abeilles (de I/I ooo à I/I5 000).

Comme on peut le remarquer sur ces deux tableaux, la concentration du sirop à I/I ooo de fluor, s'avère extrêmement toxique pour les abeilles. I,es abeilles ne survivent pas à cette dose généralement plus de 2 à 3 jours. On observe cependant dans cette série, une certaine irrégularité dans les résultats. C'est ainsi que les abeilles qui reçurent le sirop à $I / 5$ ooo de fluor moururent très rapidement et n'ont absorbé en moyenne que $\mathrm{x} 2 \gamma$ par abeille. Ce fait peut être attribué à la plus grande sensibilité de certains lots et aussi à leur affaiblissement pendant leur mise en cage. D'autre part on doit tenir compte du fait que les abeilles prélevées dans les ruches pendant l'été à la fin du printemps, sont d'âges différents ; certaines butineuses peuvent se trouver déjà au terme de leur vie au moment du prélèvement.

Néanmoins dans la série E, comme dans plusieurs des séries précédentes, les abeilles qui reçurent une dose très faible de fluor (concentration de I/ Ioo ooo vécurent aussi longtemps que les témoins et ont eu un coefficient de survie supérieur à celui des témoins tout en ayant absorbé pendant la période de leur survie $23 \gamma$ de fluor par individu.

TABLEAU 5

\begin{tabular}{|c|c|c|c|c|c|c|c|}
\hline No Expérience & $\begin{array}{l}\text { Nombre } \\
\text { d'abeilles }\end{array}$ & $\begin{array}{c}\text { Consomma- } \\
\text { tion de } \\
\text { sirop } \\
\text { (en cc) }\end{array}$ & $\begin{array}{l}\text { Concentr. } \\
\text { du sirop } \\
\text { en fluor }\end{array}$ & $\begin{array}{c}\text { Survie } \\
\text { maximum } \\
\text { (en jours) }\end{array}$ & $\begin{array}{l}\text { Survie } \\
\text { moyenne } \\
\text { (en jours) }\end{array}$ & $\begin{array}{c}\text { Consomma- } \\
\text { tion en } \\
\text { F/Ab/jour } \\
(\mathrm{en} \gamma)\end{array}$ & $\begin{array}{c}\text { Consomm. } \\
\text { tot. F/Ab } \\
\text { (surv. moy.) } \\
\text { (en } \gamma \text { ) }\end{array}$ \\
\hline $25 \ldots \ldots \ldots \ldots \ldots$ & 14 & 1 & 1) 1000 & 1 & 1 & 71 & 71 \\
\hline $26 \ldots \ldots \ldots \ldots \ldots$ & 21 & 3,8 & 1) 15000 & 7 & 2,8 & 4,2 & 12 \\
\hline $27 \ldots \ldots \ldots \ldots \ldots$ & 23 & 16,5 & 1) 20000 & 24 & 18 & 2 & 36 \\
\hline $28 \ldots \ldots \ldots \ldots$ & 26 & 15,3 & 1) 25000 & 23 & 12,1 & 1,9 & 23 \\
\hline $29 \ldots \ldots \ldots \ldots$ & 15 & 8,3 & 1) 30000 & 24 & 9,1 & 2 & 18 \\
\hline $30 \ldots \ldots \ldots \ldots$ & 23 & 19,2 & 1) 50000 & 30 & 17,5 & 0,94 & 16 \\
\hline $31 \ldots \ldots \ldots \ldots \ldots$ & 13 & 29,9 & $1 / 100000$ & 37 & 28,6 & 0,80 & 23 \\
\hline Témoin. & 16 & 37,4 & & 38 & 24,8 & & \\
\hline
\end{tabular}

TABIEAU 6

\begin{tabular}{|c|c|c|c|c|c|c|c|}
\hline No Expérience & $\begin{array}{l}\text { Nombre } \\
\text { d'abeilles }\end{array}$ & $\begin{array}{c}\text { Consomma- } \\
\text { tion de } \\
\text { sirop (en cc) }\end{array}$ & $\begin{array}{l}\text { Concentra- } \\
\text { tion du sirop } \\
\text { en fluor }\end{array}$ & $\begin{array}{c}\text { Survie } \\
\text { maximum } \\
\text { (en jours) }\end{array}$ & $\begin{array}{c}\text { Survie } \\
\text { moyenne } \\
\text { (en jours) }\end{array}$ & $\begin{array}{c}\text { Consomma- } \\
\text { tion en } \\
\text { F/Ab/jour } \\
(\text { en } \gamma)\end{array}$ & $\begin{array}{c}\text { Consomm. } \\
\text { totale F/Ab } \\
\text { (surv. moy.) } \\
(\text { en } \gamma)\end{array}$ \\
\hline $32 \ldots \ldots \ldots \ldots \ldots$ & 14 & 1,6 & 1/ 1000 & 3 & 2 & 55 & 110 \\
\hline $33 \ldots \ldots \ldots \ldots \ldots$ & 18 & 1,7 & 1) 2000 & 3 & 2,3 & 23 & 53 \\
\hline $34 \ldots \ldots \ldots \ldots \ldots$ & 7 & 1,7 & 1) 5000 & 4 & 3,1 & 15 & 48 \\
\hline $35 \ldots \ldots \ldots \ldots$ & 22 & 5,5 & $1 / 10000$ & 8 & 5 & 5 & 25 \\
\hline $36 \ldots \ldots \ldots \ldots$ & 7 & 4,4 & $1 / 15000$ & 11 & 9,3 & 4,5 & 42 \\
\hline Témoin $\ldots . . .$. & 10 & 21,4 & & 50 & 43,6 & & \\
\hline
\end{tabular}

Annales de l'Abeille. - Ig6z. 
Il ressort des essais qui composent ces deux séries que les abeilles qui moururent par suite de la concentration très forte du fluor absorbèrent dans ces expériences respectivement $25 \gamma$ et $32 \gamma, 7$ I $\gamma$ et I Io $\gamma$ par abeille, c'est-à-dire les doses qui dépassent de 12,5 à 20 fois celle qui est considérée comme léthale.

\section{$2^{\circ}$ Essais D'INTOXICATION DES ABEILI,ES PAR L'ADMINISTRATION SIMULTANÉE DE SIROP DE SUCRE PUR ET DE SIROP ADDITIONNÉ DE NaF}

\section{Série G}

Cette série d'essais diffère des précédentes en ce sens que les abeilles ont à leur disposition deux sirops : l'un normal, l'autre fluoré, à des concentrations de $I / I$ ooo, I/2 ooo, I/5 ooo et I/Io ooo, ceci afin d'établir si les insectes maintenus dans les cagettes ont une répulsion marquée pour le sirop contenant le toxique à une forte concentration.

Les essais ont été effectués au mois d'octobre et novembre du 24-Io au Io-II, à la température du laboratoire de $22-25^{\circ} \mathrm{C}$.

On constate que dans ces expériences, les abeilles se sont attaquées aussi bien au sirop contenant le fluor qu'à celui qui ne le contenait pas.

Comme dans la plupart des essais qui furent relatés plus haut, la concentration de I/I ooo de fluor s'est avérée la plus toxique et la survie des abeilles n'a pas dépassé 2 jours, pendant lesquels elles n'absorbèrent chacune que $22 \gamma$.

Par contre dans ces essais, les abeilles nourries au moyen des solutions de fluor à I/ 2 ooo, I/5 ooo et I/ /o ooo vécurent plus longtemps et ne moururent qu'après avoir absorbé 42,26 et $23 \gamma$ respectivement.

TABLEAU 7

\begin{tabular}{|c|c|c|c|c|c|c|c|c|}
\hline \multirow{2}{*}{$\begin{array}{l}\text { No Expé- } \\
\text { périence }\end{array}$} & \multirow{2}{*}{$\begin{array}{l}\text { Nombre } \\
\text { d'abeilles }\end{array}$} & \multicolumn{2}{|c|}{$\begin{array}{l}\text { Consommation de } \\
\text { sirop }\end{array}$} & \multirow{2}{*}{$\begin{array}{l}\text { Concentra- } \\
\text { tion du } \\
\text { sirop en } \\
\text { fluor }\end{array}$} & \multirow{2}{*}{$\begin{array}{c}\text { Survie } \\
\text { maximum } \\
\text { (en jours) }\end{array}$} & \multirow{2}{*}{$\begin{array}{c}\text { Survie } \\
\text { moyenne } \\
\text { (en jours) }\end{array}$} & \multirow{2}{*}{$\begin{array}{l}\text { Consomma- } \\
\text { tion en } \\
\text { F/Ab/jour } \\
(\text { en } \gamma)\end{array}$} & \multirow{2}{*}{$\begin{array}{c}\text { Consomm. } \\
\text { totale F/Ab } \\
\text { (surv.moy.) } \\
(\text { en } \gamma \text { ) }\end{array}$} \\
\hline & & $\begin{array}{l}\text { sans } \\
\text { fluor }\end{array}$ & $\begin{array}{l}\text { avec } \\
\text { fluor }\end{array}$ & & & & & \\
\hline 33. & 32 & 0,5 & 0,7 & 1/ 1000 & 2 & 2 & 11 & 22 \\
\hline $34 \ldots$ & 13 & 2,5 & 1,1 & 1) 2000 & 11 & 5,2 & 8 & 42 \\
\hline $35 \ldots \ldots \ldots$ & 40 & 5,1 & 5,3 & 1/ 5000 & 16 & 7,1 & 3,6 & 26 \\
\hline $36 \ldots \ldots \ldots$ & 8 & 1,9 & 1,9 & $1 / 10000$ & 10 & 6,7 & 3,5 & 23 \\
\hline
\end{tabular}

Il ressort ainsi de ces essais, que les doses fortes de fluor dans le sirop n'exercent pas l'effet répulsif sur les abeilles contentues dans les cagettes, et que, d'autre part, une forte concentration de fluor dans le sirop peut provoquer la mort des abeilles à une dose relativement faible.

Cette expérience gagnerait cepenđant à être répétée à la température de $32^{\circ} \mathrm{C}$. 
$3^{\circ}$ ESSAIS DE NOURISSEMENT INTERMITTENT DES ABEILIES PAR DU SIROP PUR ET DU SIROP CONTENANT DU FLUOR A I/I OOO.

\section{Série $H$}

Dans ces expériences, le sirop fluoré à I/I ooo, concentration invariablement mortelle pour les abeilles, leur est administré d'une manière discontinue pendant I heure, le $3^{\mathrm{e}}$ jour $; 2$ heures le $4^{\mathrm{e}}$ jour et 3 heures $1 \mathrm{e} 5^{\mathrm{e}}$ jour et enfin $1 \mathrm{e} \mathrm{I} 4^{\mathrm{e}}$ jour pendant 24 heures. Les abeilles reçoivent le sirop normal avant et après les doses toxiques. Ces essais ont été effectués afin de rechercher l'existence d'une éventuelle accoutumance au toxique ou bien au contraire d'une sensibilisation. Normalement, en recevant le sirop à I/I ooo, les abeilles auraient dû mourir dans les 24 heures qui suivirent l'administration de la dose. On constate, toutefois, que quelques abeilles de certains lots qui ont été remises à la consommation du sirop de sucre pur après avoir consommé à plusieurs reprises, et pour la dernière fois pendant 24 heures, du sirop contenant $\mathrm{I} / \mathrm{I}$ ooo de fluor, ont vécu très longtemps.

Sur le tableau 8 ci-dessous, figurent les résultats de ro expériences effectuées dans le but indiqué plus haut.

TABLEAU 8

\begin{tabular}{|c|c|c|c|c|c|c|c|c|}
\hline $\begin{array}{c}\text { No Expé- } \\
\text { rience }\end{array}$ & $\begin{array}{c}\text { Nombre } \\
\text { d'abeilles }\end{array}$ & $\begin{array}{l}\text { Consom- } \\
\text { mation de } \\
\text { sirop } \\
\text { (en cc) }\end{array}$ & $\begin{array}{c}\text { Consomm. } \\
\text { totale } \mathrm{F} / \mathrm{Ab} \\
\text { au } 14^{\mathrm{e}} \text { jour } \\
(\text { en } \gamma)\end{array}$ & $\begin{array}{c}\text { Nombre } \\
\text { d'abeilles } \\
\text { survivant } \\
\text { au } 14^{\mathrm{e}} \text { jour }\end{array}$ & $\begin{array}{c}\text { Consomm. } \\
\text { en } \mathrm{F} / \mathrm{Ab} \text { le } \\
14^{\mathrm{e}} \text { jour } \\
(\mathrm{en} \gamma)\end{array}$ & $\begin{array}{c}\text { Survie } \\
\text { maximum } \\
\text { après le } \\
14^{\mathrm{e}} \mathrm{j}(\mathrm{en} \mathrm{j})\end{array}$ & $\begin{array}{c}\text { Survie } \\
\text { moyenne } \\
\text { après le } \\
14^{\mathrm{e}} \mathrm{j}(\mathrm{en} \mathrm{j})\end{array}$ & $\begin{array}{l}\text { Consomm. } \\
\text { tot. F/Ab } \\
\text { survivant le } \\
14^{\mathrm{e}} \mathrm{j}(\mathrm{en} \gamma)\end{array}$ \\
\hline $37 \ldots \ldots$ & 15 & 4,4 & 43 & 2 & 100 & 4 & 2 & 143 \\
\hline $38 \ldots \ldots$ & 10 & 6,5 & 92 & 4 & 25 & 7 & 5,2 & 117 \\
\hline $39 \ldots \ldots$ & 15 & 9,6 & 47 & 5 & 40 & 21 & 11,6 & 87 \\
\hline $40 \ldots \ldots$ & 17 & 9,1 & 45 & 7 & 0 & 32 & 5,7 & 45 \\
\hline $41 \ldots \ldots$ & 9 & 1,7 & 102 & 0 & 0 & 0 & 0 & 0 \\
\hline $42 \ldots \ldots$ & 19 & 20,8 & 37 & 8 & 25 & $5 t$ & 23,7 & 62 \\
\hline 43. & 17 & 9 & 32 & 14 & 32 & 36 & 23,2 & 64 \\
\hline $44 \ldots \ldots$ & 15 & 7,6 & 33 & 8 & 31 & 31 & 22 & $6^{\prime}$ \\
\hline $45 \ldots \ldots$ & 12 & 13,3 & 30 & 8 & 25 & 22 & 15,8 & 55 \\
\hline $46 \ldots \ldots$ & 7 & 1,5 & 98 & 0 & 0 & 0 & 0 & 0 \\
\hline Témoin.. & 10 & $21,4_{t}$ & & 10 & & 36 & 30,4 & \\
\hline
\end{tabular}

Pendant les repas toxiques qui ont été accomplis par les abeilles avant le $I 4^{\mathrm{e}}$ jour, dans certaines expériences les abeilles absorbèrent de 30 à $90 \%$ de fluor et néanmoins restèrent vivantes. Après le dernier nourissement par le sirop fluoré qui eut lieu le $\mathrm{I}_{4}$ e jour, certaines abeilles des divers lots présentèrent une survie maxima qui atteignit dans un cas 54 jours et des survies moyennes de 23,7 et 23,2 jours après le repas qui aurait normalement dû leur être fatal en $24-48$ heures.

Ces expériences démontrent qu'il y a dans les cas d'un nourissement intermittent tel qu'il est exposé une nette accoutumance au fluor et l'acquisition d'une résistance contre des doses mortelles.

En effet, malgré 1'absorption par les abeilles de doses énormes de fluor allant de 50 à $140 \gamma$, leur survie est très supérieure à celle des Abeilles non préparées, soumises à des doses bien plus fortes et surtout à la concentration similaire de $\mathrm{I} / \mathrm{I}$ ooo. 
Lorsqu'on examine attentivement le tableau on remarque la similitude de survie des abeilles dans les expériences 42,43 et 44 qui vécurent respectivement 23,7 22,7 et 22 jours après le repas toxique habituellement mortel et absorbèrent respectivement pendant leur existence 62,64 et $64 \gamma$ de fluor.

Notons que ces expériences ont été effectuées pendant la période d'activité des abeilles, c'est-à-dire entre le 22-7 et le 30-9; on pouvait s'attendre à ce qu'elles soient affaiblies dans les cagettes, ce qui n'est pas le cas comme le montrent les chiffres de plusieurs expériences.

\section{$4^{0}$ EXXPÉRIENCES D'INTOXICATION DES ABEILLES PAR DES DOSES CROISSANTES DE FLUOR}

\section{Série I}

Dans ces expériences les abeilles reçurent du sirop fluoré à des concentrations croissantes, c'est-à-dire à $I / I 00$ ooo le $\mathrm{I}^{\mathrm{er}}$ jour, à $\mathrm{I} / 50$ ooo le $2^{\mathbf{e}}$ jour, à $\mathbf{I} / 30$ ooo les $3^{\mathrm{e}}$ et $4^{\mathrm{e}}$ jours, à $\mathrm{I} / 25$ ooo les $5^{\mathrm{e}}$ et $6^{\mathrm{e}}$ jours, à $\mathrm{I} / 20$ ooo les $7^{\mathrm{e}}, 8^{\mathrm{e}}$ I6 et $\mathrm{I} 7^{\mathrm{e}}$ jours, et $9^{\mathrm{e}}$ jours, à $\mathrm{I} / \mathrm{I} 5000$ les $\mathrm{IO}^{\mathrm{e}}, \mathrm{II}^{\mathrm{e}}$ et $\mathrm{I}^{\mathrm{e}}$ jours, à $\mathrm{I} / \mathrm{IO}_{0}$ ooo les $\mathrm{I} 3, \mathrm{I} 4$, I5, et à $\mathrm{I} / 5000$ les I 8, I9, 20, 2 I et $22^{\mathrm{e}}$ jours jusqu'à la fin de l'expérience qui a débuté le I-5 et fut terminée le $28-5$.

TABLEAU 9

\begin{tabular}{|c|c|c|c|c|c|c|}
\hline No Expérience & $\begin{array}{l}\text { Nombre } \\
\text { d'abeilles }\end{array}$ & $\begin{array}{l}\text { Consomma- } \\
\text { tion de sirop } \\
(\text { en cc) }\end{array}$ & $\begin{array}{c}\text { Survie } \\
\text { maximum } \\
\text { (en jours) }\end{array}$ & $\begin{array}{c}\text { Survie } \\
\text { moyenne } \\
\text { (en jours) }\end{array}$ & $\begin{array}{c}\text { Consomma- } \\
\text { tion en } \\
\text { F } / \mathrm{Ab} / \mathrm{j}(\mathrm{en} \gamma)\end{array}$ & $\begin{array}{c}\text { Consomm. } \\
\text { tot. F/Ab } \\
\text { (Surv. moy.) } \\
\text { (en } \gamma \text { ) }\end{array}$ \\
\hline $47 \ldots \ldots \ldots \ldots \ldots$ & 7 & 10,4 & 22 & 17 & 5,8 & 98 \\
\hline $48 \ldots \ldots \ldots \ldots \ldots$ & 10 & 10,3 & 21 & 16,8 & 3 & 50 \\
\hline $49 \ldots \ldots \ldots \ldots \ldots$ & 18 & 13,4 & 21 & 17 & 2 & $3 / t$ \\
\hline $50 \ldots \ldots \ldots \ldots$ & 14 & 7,3 & 10 & 8,1 & 1,5 & 12 \\
\hline $51 \ldots \ldots \ldots \ldots$ & 13 & 7,9 & 16 & 8,6 & 2 & 17 \\
\hline $52 \ldots \ldots \ldots \ldots \ldots$ & 14 & 6,6 & 8 & 8 & 1,5 & 12 \\
\hline $53 \ldots \ldots \ldots \ldots \ldots$ & 10 & 9,8 & 19 & 15,2 & 2,8 & 43 \\
\hline $54 \ldots \ldots \ldots \ldots \ldots$ & 11 & 7,7 & 11 & 11 & 2 & 22 \\
\hline Témoin .... & 7 & 10,6 & 27 & 19,4 & & \\
\hline
\end{tabular}

Ces expériences ont eu pour but de mettre en évidence une éventuelle accoutumance des abeilles au toxique administré à doses croissantes et, d'autre part, de voir leur comportement dans ces conditions comparativement à celui des abeilles témoins et de celles qui reçurent toujours une dose égale de fluor.

On constate que les résultats ne sont pas similaires dans toutes les expériences mais un fait ressort néanmoins clairement, c'est que les abeilles, en recevant des doses croissantes de fluor, présentent une survie de 17 , I6, 8 et I7 jours et sont capables d'absorber $98 \gamma, 5^{\circ} \gamma$ et $34 \gamma$ de fluor respectivement. Il est intéressant aussi de noter que les abeilles qui reçurent le sirop à la concentration de $\mathrm{I} / 5$ ooo defluor ont, survécu plus longtemps (à juger des résultats plus détaillés, non rapportés sur ce tableau) que celles qui étaient intoxiquées directement par le sirop à $\mathbf{r} / 5$ ooo de fluor. 


\section{$5^{\circ}$ ESSAIS D'ADMINISTRATION AUX ABEILLES DE CONCENTRATIONS FAIBLES DE FLUOR SUIVIE D'ALIMENTATION BRUSQUE PAR UN SIROP A CONCENTRATION FORTE ET GÉNÉRALEMENT MORTELILE (I/I OOO)}

\section{Série J}

Dans cette série composée de trois expériences, les abeilles ont reçu du sirop fluoré à $I / 500$ ooo les 5 premiers jours, du sirop à $I / 250$ o0o les 3 jours suivants et à partir du 8 e jour le sirop à $\mathrm{I} / \mathrm{I}$ ooo.

Il ressort de ces expériences que les abeilles vécurent jusqu'au $8^{\mathrm{e}}$ jour sans qu'on ait observé aucune mortalité en absorbant avec leur nourriture $2 \mathrm{I}, 30-2$ et 38-2 de fluor par individu.

Ayant reçu le 8 e jour le sirop à I/I ooo de fluor les abeilles moururent toutes en moins de 48 h et en absorbant à peine $I ~ \gamma$ de fluor de plus.

TABLEAU IO

\begin{tabular}{|c|c|c|c|c|c|c|c|c|}
\hline $\begin{array}{c}\text { No Expé- } \\
\text { rience }\end{array}$ & $\begin{array}{c}\text { Nombre } \\
\text { d'abeilles }\end{array}$ & $\begin{array}{l}\text { Consom- } \\
\text { mation de } \\
\text { sirop } \\
\text { (en cc) }\end{array}$ & $\begin{array}{c}\text { Consomma- } \\
\text { tion } \\
\text { totale } \mathrm{F} / \mathrm{Ab} \\
\text { au } 8 \text { e jour } \\
\text { en }(\gamma)\end{array}$ & $\begin{array}{l}\text { Nombre } \\
\text { d'abeilles } \\
\text { survivant } \\
\text { au } 8^{\mathrm{e}} \text { jour }\end{array}$ & $\begin{array}{l}\text { Consom- } \\
\text { mation } \\
\text { en } \mathrm{F} / \mathrm{Ab} \text { le } \\
8^{\mathrm{e}} \text { jour } \\
(\mathrm{en} \gamma)\end{array}$ & $\begin{array}{l}\text { Survie } \\
\text { maximum } \\
\text { après le } \\
8^{\mathrm{e}} \text { jour } \\
\text { (en jours) }\end{array}$ & $\begin{array}{l}\text { Survie } \\
\text { moyenne } \\
\text { après le } \\
8^{e} \text { jour } \\
\text { (en jours) }\end{array}$ & $\begin{array}{l}\text { Consomm. } \\
\text { totale F/Ab } \\
\text { survivant le } \\
8^{\text {e jour }} \\
(\text { en } \gamma \text { ) }\end{array}$ \\
\hline $\begin{array}{l}55 \ldots \ldots \\
56 \ldots \ldots \\
57 \ldots \ldots\end{array}$ & $\begin{array}{l}10 \\
17 \\
11\end{array}$ & $\begin{array}{l}4,8 \\
6,4 \\
5,6\end{array}$ & $\begin{array}{l}1 \\
0,8 \\
0,7\end{array}$ & $\begin{array}{l}10 \\
17 \\
11\end{array}$ & $\begin{array}{l}21 \\
30,2 \\
28,2\end{array}$ & $\begin{array}{l}2 \\
1 \\
2\end{array}$ & $\begin{array}{l}1,9 \\
1 \\
1,9\end{array}$ & $\begin{array}{l}22 \\
31 \\
29\end{array}$ \\
\hline $\begin{array}{l}\mathrm{T} 1 \ldots . . \\
\mathrm{T} 2 \ldots .\end{array}$ & $\begin{array}{l}22 \\
16\end{array}$ & $\begin{array}{l}31,3 \\
33\end{array}$ & & $\begin{array}{l}20 \\
16\end{array}$ & & $\begin{array}{l}55 \\
47\end{array}$ & $\begin{array}{l}12,8 \\
32\end{array}$ & \\
\hline
\end{tabular}

I1 paraît ainsi que la préparation des abeilles par l'absorption de doses très faibles n'aboutit pas à leur protection, contrairement avec ce qu'on constate en leur administrant pendant 3 courtes périodes une dose forte de fluor, puis cette même dose pendant une période de 24 heures (voir tableau 8 ).

\section{DISCUSSION E'T CONCLUSIONS}

Les résultats de ce travail ne peuvent être comparés avec ceux qui ont été obtenus antérieurement par d'autres auteurs et par nous-mêmes car dans ce travail, la technique utilisée était différente de celles employées antérieurement.

Il ressort des expériences entreprises que le fluor, sous forme de $\mathrm{NaF}$ en solution dans le sirop de sucre administré aux abeilles placées dans les cagettes, s'avère toxique à une concentration de $\mathrm{I} / \mathrm{I}$ ooo de fluor. Concentration qui nous paraît difficilement réalisable dans la nature. Cette toxicité se manifeste lorsque le sirop à cette concentration est administré continuellement pendant 24 heures ou plus et se traduit par la mort de toutes les abeilles. 
Ia toxicité du sirop fluoré devient de moins en moins importante avec des solutions plus étendues de $\mathrm{NaF}$; et celle de $\mathrm{I} / 5$ ooo constitue la limite de la toxicité réelle du fluor.

L'effet des solutions à I/50 o0o et $\mathrm{I} /$ roo ooo est pratiquement nul.

Les abeilles qui absorbent le fluor à $\mathrm{I} / 100000$, et parfois à $\mathrm{I} / 50000$, ont vécu dans certaines expériences aussi longtemps et même plus longtemps que les témoins tout en ayant absorbé des quantités de fluor nettement supérieures à celle qui est considérée par divers auteurs comme une dose léthale. A juger de certaines expériences, le fluor en concentration très faible ( $\mathbf{r} /$ IOO OOO) n'agit pas comme toxique mais renforce la vitalité des abeilles en captivité. Le fluor en quantités minimes agit peut-être en tant que catalyseur de certains processus physiologiques chez les abeilles maintenues dans les cagettes expérimentales. Il n'est pas à exclure qu'à ces concentrations faibles le fluor atténue l'auto-intoxication possible des abeilles par les excréments qui s'accumulent dans l'ampoule rectale en agissant sur les bactéries de putréfaction.

L'évaluation du taux moyen de fluor absorbé par les abeilles individuellement (pendant la période de leur survie, et après leur mort), a permis de constater que ces insectes peuvent avant de mourir, et même bien longtemps avant leur mort, absorber une quantité de fluor triple, quatruple et même dix fois plus élevée que celle considérée par certains auteurs, comme dose mortelle.

Le nourrissement des abeilles par le sirop à concentration de fluor d'abord très faible, puis progressivement de plus en plus forte, n'a pas complètement annihilée l'effet de la dose forte (en l'occurence de $1 / 5000$ ), mais les abeilles, ainsi traitées, ont montré dans certaines expériences une survie maxima et moyenne assez grandes tout en ayant absorbé des quantités de fluor considérables, plus précisément 34, 50 et $98 \gamma$ de fluor pour un survie moyenne de $I_{7}$ jours.

Quelques essais ont consisté en l'administration aux abeilles de doses de fluor très faibles (I/500 000, I/250 000) avant leur intoxication par the dose forte et, mortelle de I/I ooo. Les abeilles moururent dans un délai de $24 \mathrm{~h}$ ce qui dénote que l'absorption du sirop à faible concentration de fluor ne les a pas protégées contre l'intoxication par la dose forte.

Par contre, nous avons pu constater que dans d'autres circonstances on peut obtenir une résistance des abeilles contre la dose mortelle. C'est ainsi qu'en administrant à certains lots d'abeilles, d'abord le sirop de sucre pur, puis ensuite, pendant I, 2 ou 3 heures les $3^{\mathrm{e}}, 4^{\mathrm{e}}$ et $5^{\mathrm{e}}$ jours respectivement la concentration mortelle de sirop à $I / I$ ooo de fluor, nous n'avons obtenu jusqu'au $\mathrm{I}_{4}{ }^{\mathrm{e}}$ jour qu'une mortalité partielle des abeilles bien que certaines aient absorbé, d'après les calculs effectués de 30 à $92 \gamma$ de fluor.

Les abeilles qui ont survécu ont reçu le sirop concentré le $\mathrm{I}_{4}^{\mathrm{e}}$ jour et pendant 24 heures, c'est-à-dire le temps suffisant pour provoquer la mort des abeilles non préparées.

Elles ont vécu pendant un certain temps encore et ne sont mortes qu'après un temps qui correspondait à la survie maxima et à la survie moyenne des témoins et après avoir absorbé pendant cette survie des doses aussi élevées de fluor que 45 et I $43 \gamma$.

On doit conclure ainsi que, même à une concentration très forte et mortelle pour les abeilles, l'effet dı fluor est atténué et đans certains cas est même annihilé 
lorsqu'il est administré à des fortes doses d'une manière brève et intermittente avec le sirop de sucre.

Il n'est pas à exclure ainsi que le nourissement des abeilles au printemps par le sirop fluoré dans les conditions appropriées pourrait atténuer l'intoxication des abeilles par le fluor, cette intoxication nous paraissant cependant sujette à caution étant donné que les concentrations mortelles de fluor pour les abeilles dans les conditions expérimentales sont très élevées et ne sauraient certainement se réaliser dans les conditions naturelles.

Néanmoins, il faut continuer les recherches dans ces directions et reconnaittre l'effet du fluor sur les abeilles qu'il soit additionné de sirop ou de miel, de pollen ou de sucre candi, etc.

Ce sont là des travaux qui feront l'objet de nos publications ultérieures.

Reçu pour publication en juillet 1962.

\section{SUMMARY}

EXPERIMENTAL RESEARCH ON THE TOXICITY OF FLUORINE TO BEES

The experiments described in this paper have shown that fluorine (in the form of a solution $\mathrm{NaF}$ in sugar syrup) was toxic to bees only at concentrations too high to occur under natural conditions.

Bees fed on a syrup containing a low concentration of fluorine could ingest more than what some authors considered to be lethal doses of the latter and yet gave the same survival figures as the controls.

One of the feeding methods described enabled it to be stated that bees might be inured to doses of fluorine which under normal conditions would prove fatal.

\section{RÉFÉRENCES BIBL,IOGRAPHIQUES}

BEI-BIF_ko G. I. et al., I955. Entomologie agricole (en russe) Selshosgis. Mloscou-Leningrad.

Borchert II., I953. Zur geochimie des Fluors. Meidelberger Beitr. Mineralog. Pelrogr. Berlin-(iöttingen, 3, $36-+3$.

Bredenani G., Radeloff, I939. Bienensterben durch Auorhältige Fabrikalgase. Deutscher Imkerführer. Berlin $\mathbf{H} 2,59^{-61}$.

BredenanN G., I956. Biochimie und Physiologie des Fluors $2^{\mathrm{e}}$ Auflage, Akademie Verlag.

Dictionnaire d'information de l'Apiculteur. (Slovar Spravotchik Ptchelovoda) i955.

GoETzE G., r932. Zwai neue methoden zum quantitativen Studium der (iftigheit von Insectiziden. Anz. Schädlingsk., 8, (5), 54.57.

Guinos J., 1956. Intoxication expérimentale des Abeilles par divers dérivés fluorés. Bull. Acad. Vétér., $29,4+16$

Jacimonicz Th., Tounanoff C., Wemer F., i958. Le fluor chez les Abeilles dans la Haute-Autriche. Rev. Frane. Apic.

Kuxze, G., I929. Geschmäks und Giftwirkung des Fluomatriums auf die Honigbiene. Nachricht. Deutsch. Pllant3enschtzd. 9, $\mathbf{H}$ 2, I 3 -I4.

Maurizio A., 1955. Pflanzenschutzmittel und Industrieabgase als Ursache von Isienenvergiftung. Schweitz. Landie. Monxtshefte, 485 .

Maurizio A., I 956. Protokoll der Internationalen Koferenz zur Koordinirung der Eischätszung der Bienengefärlichkeit von Pflanzenschtzmitteln, 14. 
Maturizio A., I957. Bestimming der letalen Dosis iniger Fluorverbindung en für Bienen. Zugleich ein Betrag zur Methodik der Giftwerbestimmung in Bienenversuchen. Verhand. des 4 Inter. Ptlanzenschutz-Kong, Bd. 2, S I 709-1 7 I 3 .

Maurizio A., STAub M., I956. Bienenvergiftungen mit fluorhaltigen Industrie Abgasen. Schweit». Bienen. Zeitg., 79, 476-486.

Rousseau M., I954. Exposé au cours de l'Assemblée générale de l'U. N. A. F., le 7 novembre I954. Rev. Franc. Apic., 3, r 5 5.

Rousseau M., 195\%. Recherches sur la "doje léthale médiane " du fluor pour l'Abeille. Rez. Franc. Apic. (1 29), 22.

Toumanoff C., r95r. Les maladies des Abeilles. No spécial, Rev. Franc. Apic., n 68, 325.

ToumanofF C., Jachrmowicz Th., 1959. Observations au sujet de l'action du fluor sur les Abeilles. $C . R$. Acad. Sci., 249.

Toumanoff C., Jacinmowicz Th.. Ig6o. L'action dufluor sur les Abeilles. Rev. Franc. Apic., 166, I 58-162. Toumanoff C.. 196r. Étude de l'action du fluor sous forme de NaF sur les colonies d'Abeilles. Bull. Apic., 2. 223-24s. 